ARTICLE

DOI: $10.1038 /$ s41467-018-05751-y

\title{
Surveying silicon nitride nanopores for glycomics and heparin quality assurance
}

Buddini Iroshika Karawdeniya', Y.M. Nuwan D.Y. Bandara ${ }^{1}$, Jonathan W. Nichols ${ }^{1}$, Robert B. Chevalier ${ }^{1} \&$ Jason R. Dwyer (1) ${ }^{1}$

Polysaccharides have key biological functions and can be harnessed for therapeutic roles, such as the anticoagulant heparin. Their complexity-e.g., >100 monosaccharides with variety in linkage and branching structure-significantly complicates analysis compared to other biopolymers such as DNA and proteins. More, and improved, analysis tools have been called for, and here we demonstrate that solid-state silicon nitride nanopore sensors and tuned sensing conditions can be used to reliably detect native polysaccharides and enzymatic digestion products, differentiate between different polysaccharides in straightforward assays, provide new experimental insights into nanopore electrokinetics, and uncover polysaccharide properties. We show that nanopore sensing allows us to easily differentiate between a clinical heparin sample and one spiked with the contaminant that caused deaths in 2008 when its presence went undetected by conventional assays. The work reported here lays a foundation to further explore polysaccharide characterization and develop assays using thin-film solidstate nanopore sensors.

\footnotetext{
${ }^{1}$ Department of Chemistry, University of Rhode Island, 140 Flagg Road, Kingston 02881, USA. Correspondence and requests for materials should be addressed to J.R.D. (email: jason_dwyer@uri.edu)
} 
O ligo- and polysaccharides are ubiquitous in nature, with a broad spectrum of roles that includes energy-storage and provision (including as a foodstuff), structural building block (e.g., cellulose), therapeutic function (e.g., the anticoagulant heparin), and a vital part in biological recognition processes ${ }^{1-11}$. Conventional chemical analysis tools are frequently challenged by the daunting complexity of polysaccharide analysis: ${ }^{12,13}$ identification of monomer composition ( 120 naturally occurring monomers!) and sequence, monomer linkage types, stereochemistry, polymer length, and degree of polymer branching ${ }^{13}$. These challenges were tragically driven home in 2008 when undetected contamination of the common anticoagulant heparin by a structurally similar adulterant, oversulfated chondroitin sulfate (OSCS), resulted in profoundly adverse clinical consequences in the United States, including $\sim 100$ deaths ${ }^{14-19}$. Glycan samples can be challenged by heterogeneity and low abundance in addition to chemical and structural diversity, so while new analysis tools have been broadly called for ${ }^{12,13,20}$, single-molecule-sensitive methods are a particularly compelling goal for glycomics-more so given the absence of sample amplification techniques analogous to PCR for DNA sequencing $^{21}$. Nanopore single-molecule methods have emerged as a powerful tool for characterizing DNA and proteins, including aspects of sequence, structure, and interactions ${ }^{22-28}$. Monomerresolved length determinations of more prosaic polyethylene glycol samples further buttress the potential of suitably configured nanopore assays for the analysis of polymers with biological utility $^{29}$. The simplest implementation for nanopore measurements places the nanopore- $\mathrm{a}<100 \mathrm{~nm}$-long nanofluidic channel through an insulating membrane-between two electrolyte solutions (Fig. 1). Ion passage through the nanopore in response to a voltage applied across the pore gives the baseline open pore current, $i_{0}$; passage of a molecule into, across, or through the nanopore disrupts this ion flow to give a blocked-pore current, $i_{\mathrm{b}}$. A discernible current perturbation reveals the presence of an analyte, and the sign, magnitude, and temporal structure of $i_{\mathrm{b}}$ depend strongly on size and shape of the analyte-and of the nanopore-and on the applied voltage and bulk and interfacial charge distributions. It thus provides insight into analyte presence, identity, and properties, including interactions between the analyte and pore interior or surface ${ }^{29-32}$. Analysis of the resistivepulse characteristics of a sample offers the potential to glean molecular-level insights, but the $i_{\mathrm{b}}$ characteristics can also be used more simply as benchmarks in quality assurance assays where atypical $i_{\mathrm{b}}$ values signal sample impurities.

Much groundwork must be laid, including proof-of-principle experiments, if nanopore methods are to emerge as a tool for glycan profiling-and by extension as a tool for-omics writ-large (spanning genomics, proteomics, and glycomics). Sugar-pore binding, polysaccharides, and enzyme-digested oligosaccharides

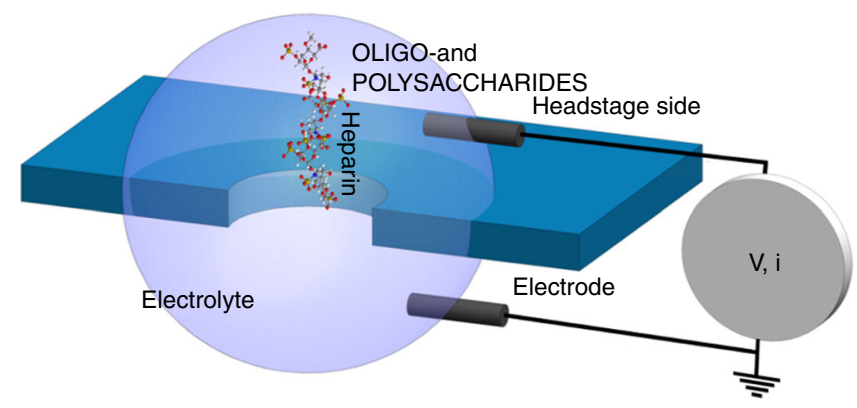

Fig. 1 Schematic of the nanopore setup. Voltage-driven passage of a molecule into, across, or through an electrolyte-filled nanopore can be used for analyte detection and characterization have been detected using a range of different nanopore platforms from protein to solid-state ${ }^{33-43}$. While solid-state nanopores in thin $(\sim 10 \mathrm{~nm})$ membranes have been often portrayed as the preeminent nanopore platform, their use to profile classes of molecules beyond DNA and proteins is in its infancy. These nanopores can be size-tuned ${ }^{44}$ to match analyte dimensions (especially relevant for branched polysaccharides), and when fabricated from conventional nanofabrication materials such as silicon nitride $\left(\mathrm{SiN}_{\mathrm{x}}\right)^{45,46}$, offer resistance to chemical and mechanical insult alongside low barriers to large-scale manufacturing and device integration. The potential for integration of additional instrumentation components, such as control and readout electrodes, around the thin-film $\mathrm{SiN}_{\mathrm{x}}$ nanopore core, is especially compelling $28,45,46$. Recent (nanopore-free) work on recognition electron tunneling measurements on polysaccharides, for example, has reaffirmed the importance of a nanopore development path that values augmented nanopore sensing capabilities ${ }^{47}$. A key question concerning the use of $\mathrm{SiN}_{\mathrm{x}}$ nanopores for polysaccharide sensing is whether this fabrication material is compatible with sensing glycans, which can exhibit a wide range of chemical and physical properties. The often challenging surface chemistry of $\mathrm{SiN}_{\mathrm{x}}$ (giving rise to a complex surface charge distribution $)^{45,46,48}$ may lead to analyte-pore interactions that hinder or prevent its use. Variability in polysaccharide electrokinetic mobility arising from differences in molecular structures may exacerbate the effect of these interactions. These issues become particularly important when analyte translocation through a constricted pore is required, such as in transverse electron tunneling measurements ${ }^{28,47}$.

Naturally occurring sodium alginate, with uses in biomedical and food industries, presents an overall negative, but unexceptional, formal charge in neutral $\mathrm{pH}$ aqueous solutions. Sourcing variability for alginates that are extracted from seaweed can be as prosaic as molecular weight to more enticing changes in the relative abundances of alginate's constituent mannuronate $(\mathrm{M})$ and guluronate $(\mathrm{G})$ residues $^{49}$. At a compositional extreme, heparin, the prevalent anticoagulant drug, is the most highly negative charge-dense biological molecule known ${ }^{50}$. This exceptional charge density couples with the demonstrated difficulty, by other methods, of detecting the negatively charged OSCS (molecular weight $\sim 17 \mathrm{kDa}^{51}$ ) contaminant in a heparin sample ${ }^{14-17}$ to make the analysis of heparin $(\sim 16 \mathrm{kDa})$ and OSCS by nanopore a compelling experimental test with clinical relevance.

The aims of the present work are: (1) to explore using $\mathrm{SiN}_{\mathrm{x}}$ nanopores for sensing polysaccharides with a range of possibly challenging chemical and physical properties; and (2) to gauge the prospects of a clinically relevant assay to detect the toxic OSCS impurity in heparin. We profile alginates with different properties -A1 $\left(M_{n} \sim 74 \mathrm{kDa}\right)$ and $\mathbf{A 2}\left(M_{n} \sim 18 \mathrm{kDa}\right)$-to diversify the analyte scope, and change the solution $\mathrm{pH}$ to explore the effect of $\mathrm{SiN}_{\mathrm{x}}$ nanopore surface charge on the electrokinetics of polysaccharide detection. Highly charge-dense heparin further expands the molecular pool, and a change of electrolyte concentration is used to improve the signal levels. Under these solution conditions, we perform analytical determinations of heparin at clinically relevant concentrations and detect OSCS impurities in the heparin sample. Using a simple statistical thresholding algorithm, we detect this impurity using nanopores differing in apparent diameter by as much as $50 \%$.

\section{Results}

Exploring nanopore polysaccharide sensing using alginates. Introduction of anionic alginate $\mathbf{A 1}\left(M_{n} \sim 74 \mathrm{kDa}\right.$; alginate masses determined by viscosity, see Supplementary Methods) into the headstage sample well failed to generate detectable transient 


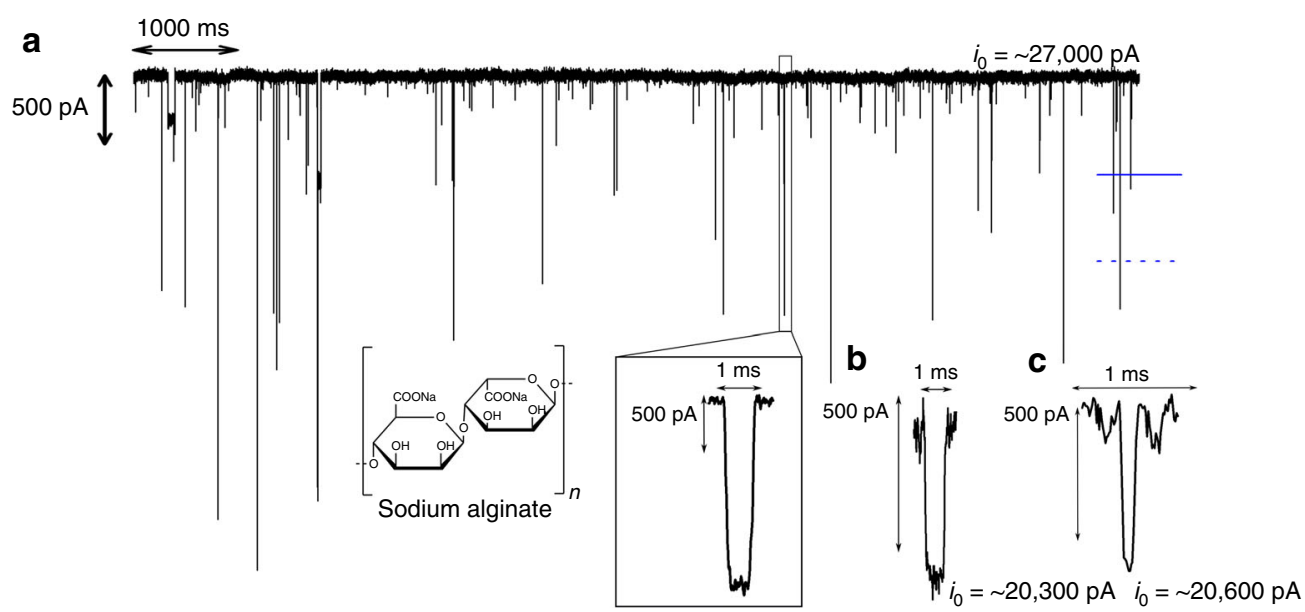

Fig. 2 Representative nanopore current trace and events from sodium alginate samples from two different sources. a A representative segment of an A1induced current trace using a $21 \mathrm{~nm}$-diameter pore; the solid blue line marks the most frequent event level, $i_{b}$, and the blue dashed line is its mean across all events. The magnified current event is from the same trace. $\mathbf{b} \mathbf{A 2}$ - and $\mathbf{c}$ enzyme-digested-A2-associated single events through a $22 \mathrm{~nm}$-diameter pore. All currents were measured in response to a $-200 \mathrm{mV}$ voltage difference

current changes when a positive voltage difference (the polarity consistent with purely electrophoretic motion for an anionic analyte) was applied with the analyte in the same well (Fig. 1). Application of a negative potential difference, instead, generated transient current changes (here denoted events) that could be readily differentiated from the open current noise with $\sim 60: 1$ event-to-noise frequency compared to analyte-free scans. Figure 2 shows a representative time trace of $\mathbf{A 1}$-induced events, with a characteristic event magnified. The frequency of discrete current blockages associated with the addition of Al showed a linear increase with analyte concentration (Supplementary Fig. 1), so that regardless of mechanism, with appropriate measurement conditions, the event frequency can be used to determine the analyte concentration. The mechanism of A1-induced signal generation was investigated in a series of experiments. Using a setup (Supplementary Fig. 2) that physically separated electrodes and nanopore, events were only detected when $\mathbf{A l}$ was injected into the well proximal to the nanopore, thus supporting a signal generation mechanism involving interaction with the nanopore and not with the electrodes. This result did not, however, distinguish between passage-free collision with the nanopore opening (bumping or blocking) or translocation through the pore ${ }^{32}$. Either mechanism (including extending the idea of bumping or blocking to allow for transient interactions of the analyte with the pore mouth), though, has the potential to deliver analytically useful sensing performance. Low analyte concentrations challenge the direct investigation of polysaccharide translocation through small, single nanopores. In one experiment to investigate this, a solution of A1 was added to the headstage side of a $\sim 22 \mathrm{~nm}$ diameter nanopore and was left overnight with a $-200 \mathrm{mV}$ voltage difference. The initially analyte-free contents of the groundstage side were then transferred to the headstage side of a fresh $\sim 17 \mathrm{~nm}$-diameter pore, and an appreciable number of A1-characteristic events (182 in $1 \mathrm{~h}$ ) were detected again using a $-200 \mathrm{mV}$ difference. Acid digestion was used as a signal generation and amplification technique (complete details in the Supplementary Methods) to convert A1 polymers to many smaller fragment-derived species absorbing at $\sim 270 \mathrm{~nm}^{52,53}$. This spectrophotometric assay (Supplementary Fig. 3) was used to confirm translocation of polysaccharide through an $\sim 8 \mathrm{~nm} \mathrm{SiN}_{\mathrm{x}}$ nanopore.

The analyte-induced translocation blockage current, $i_{\mathrm{b}}$, is expected to be determined by the properties of the analyte and its size relative to the nanopore, among other experimental factors (including interfacial phenomena) ${ }^{30,32}$. For each individual current blockage, we calculated the blockage duration, $\tau$, and the fractional blockage current magnitude, $f_{\mathrm{b}}=\left\langle i_{\mathrm{b}}\right\rangle /\left\langle i_{0}\right\rangle$, where $\langle\ldots\rangle$ denotes a time-average, and $i_{0}$ is the current through the pore when unobstructed by analyte. Plots of number of events as a function of $\tau$ and $f_{\mathrm{b}}$ (Fig. 3) provide an overarching summary of the total current trace. Given detectable differences as a function of analyte, such plots and other representations have the potential to function as analyte fingerprints in quality assurance assays. Fingerprints for $\mathbf{A 1}$ are shown in Fig. 3, acquired in $1 \mathrm{M} \mathrm{KCl}, \mathrm{pH}$ $\sim 7$ solutions using a $-200 \mathrm{mV}$ voltage difference. Supplementary Figs. 4-6 provide alternative presentations of the experimental measurements. The (most frequent) $f_{\mathrm{b}}$ increased in magnitude with increasing nanopore radius, $r_{\text {pore }}$ (that is, the relative magnitude of the current perturbations due to the analyte were reduced). This parallels the behavior observed in studies of DNA translocation that could be described using a simple volumeexclusion framework: $r_{\text {analyte }}^{2} / r_{\text {pore }}^{2}=1-f_{b}$. While nanopore diameters are fixed once fabricated (absent etching), a conformationally flexible macromolecule can present a range of apparent cross-sections to a nanopore, down to its molecular cross-section if linearized by a sufficiently small nanopore. Translocation of hyaluronic acid through $\mathrm{SiN}_{\mathrm{x}}$ nanopores fabricated using a helium ion microscope calibrated to produce $\sim 6.5-8.6 \mathrm{~nm}$ diameters yielded $f_{\mathrm{b}} \sim 0.95$. Quasi-monodisperse samples gave single-level blockages with magnitudes of $\left(1-f_{\mathrm{b}}\right)$, and multi-level blockages with magnitudes equal to $\left(1-f_{\mathrm{b}}\right)$ and approximately twice that value, indicating the presence of linear and partially folded-over biopolymers, respectively ${ }^{43}$. Nanopore geometric constraints can thus affect the effective end-to-end length of the translocating molecule or, depending on the nature of the analyte, expose surface chemistry that can similarly affect translocation times. In Fig. $3 \mathrm{~d}$, the use of a $\sim 5 \mathrm{~nm}$-diameter nanopore broadened the distribution of $f_{\mathrm{b}}$ and produced deeper blockages with longer durations than when using the larger nanopore. Lowering the electrolyte concentration can have a dramatic effect on nanopore sensing, through changes in the bulk and at interfaces. For example, reducing the ion concentration from 1 to $0.1 \mathrm{M} \mathrm{KCl}$ increases the Debye layer thickness changing the electrostatic size of the pore with consequences for electrokinetic phenomena, and electroosmosis especially. Comparing Fig. 3a, e, this change of concentration did not affect the voltage polarity 

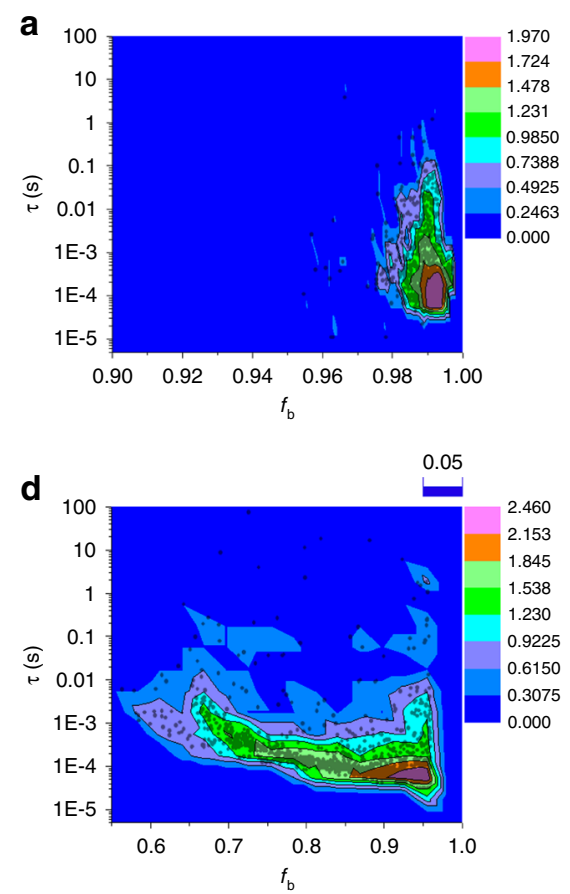
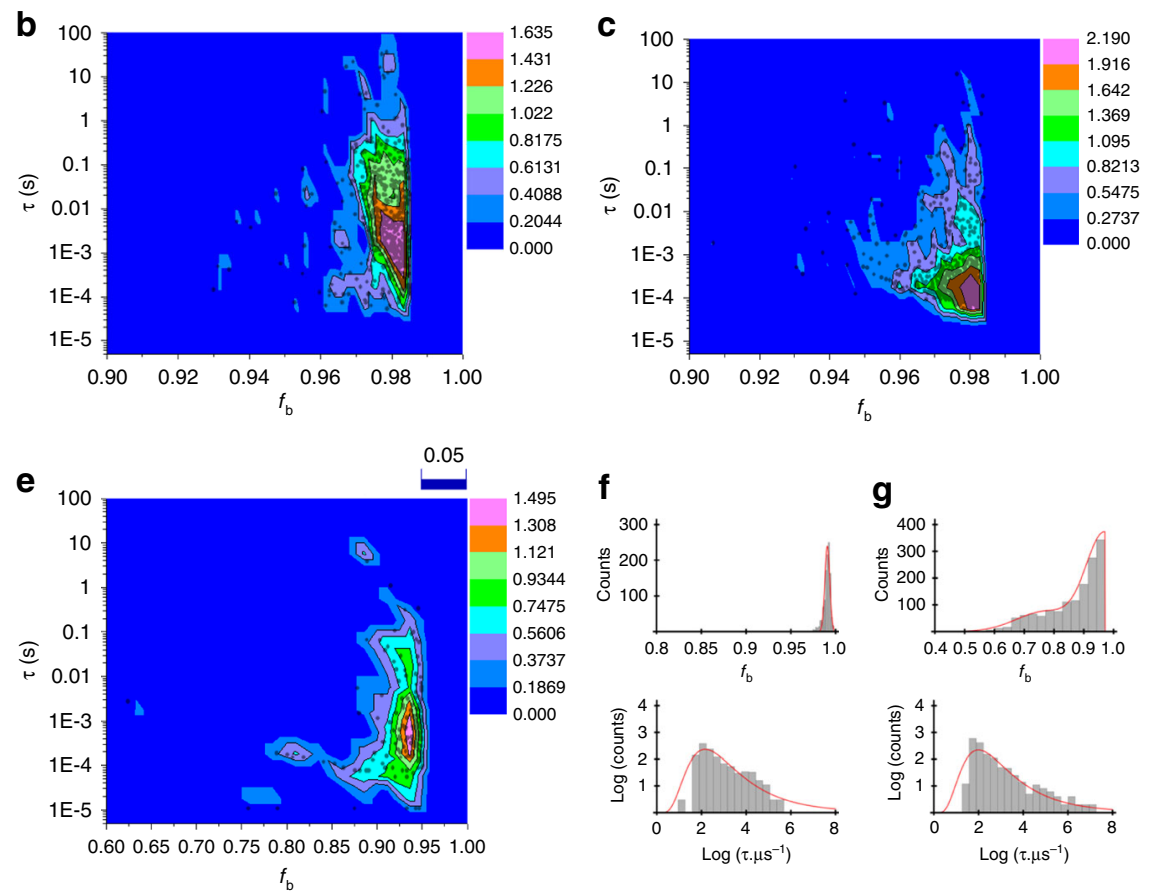
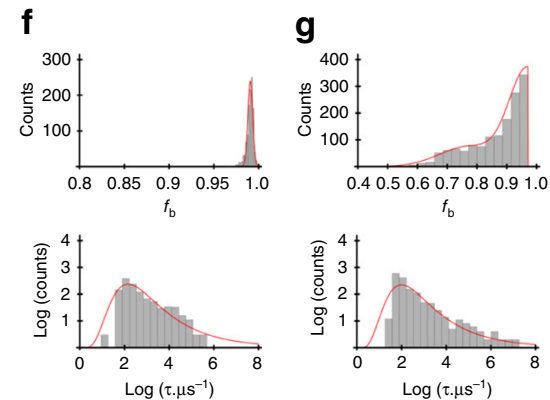

Fig. 3 Heat maps with superimposed scatter plots of alginate-induced events. Event counts (plotted as $\log _{10}$ on the color axis) of a $4 \mu \mathrm{L} 0.2 \%(\mathrm{w} / \mathrm{v})$ A1 using a $19 \mathrm{~nm}$ diameter pore $\left(\sim 0.321\right.$ events $\left.\cdot \mathrm{s}^{-1}\right)$, b $20 \mu \mathrm{L}$ of $3 \%(\mathrm{w} / \mathrm{v})$ A2 using a $\sim 21 \mathrm{~nm}\left(\sim 0.046\right.$ events. $\left.\mathrm{s}^{-1}\right)$ and $\mathbf{c} 20 \mu \mathrm{L}$ of $10-$ minute enzyme digested $3 \%(\mathrm{w} / \mathrm{v})$ A2 using a $\sim 22 \mathrm{~nm}$ diameter pore $\left(\sim 0.112\right.$ events $\left.\cdot \mathrm{s}^{-1}\right)$, all in $\mathrm{pH} \sim 7$ buffered $1 \mathrm{M} \mathrm{KCl}$. The experiment in a was repeated $\mathbf{d}$ using a $\sim 5 \mathrm{~nm}$ nanopore $\left(\sim 0.403\right.$ events $\left.\cdot \mathrm{s}^{-1}\right)$, and $\mathbf{e}$ an $\sim 18 \mathrm{~nm}$-diameter pore, but in $0.1 \mathrm{M} \mathrm{KCl}$ (vs. $1 \mathrm{M} \mathrm{KCl}$ in a) electrolyte buffered at pH $\sim\left(\sim 0.0527\right.$ events $\left.\cdot \mathrm{s}^{-1}\right)$. The plots in $\mathbf{f}$ and $\mathbf{g}$ show histograms of the data (the red lines show fits to the data) in a and $\mathbf{d}$, respectively, with additional plots in Supplementary Fig. 4 . The applied voltage difference was $-200 \mathrm{mV}$ for all measurements

needed to generate events, but decreased the $f_{\mathrm{b}}$ for the same experimental configuration, and appreciably lengthened the (most frequent) blockage duration. More profoundly, the tenfold salt concentration decrease reduced the frequency of events sixfold in the same size $\sim 18 \mathrm{~nm}$-diameter pore. We found, and exploited in a more general context for the sensing of heparin and OSCS (below), that such a simple change of electrolyte concentration is a powerful parameter for tuning our ability to sense polysaccharides. Changing the electrolyte $\mathrm{pH}$ offers a similar parameter for tuning the sensing performance of nanopores with ionizable surface groups. The surface charge of $\mathrm{SiN}_{\mathrm{x}}$ nanopores can be tuned from negative through its isoelectric point $(\sim 4.4 \pm 0.3$; standard deviation across three pores) to positive ${ }^{45,54}$, and the consequence of this $\mathrm{pH}$ change is seen in Supplementary Fig. 7: the voltage polarity for signal generation is opposite at $\mathrm{pH} 3$ and 5 (and opposite to the electrophoretic direction for all $\mathrm{pH}$ values), and the event frequency is at its minimum nearest the isoelectric point and increases with increase and decrease in $\mathrm{pH}$ from this point.

After the initial exploratory and proof-of-principle experiments using $A 1$, we turned to the second sodium alginate sample, A2, obtained from a separate supplier. In general, the interplay between analyte charge density, monomer chemical nature and polymer linkages, and electrolyte composition, is expected to influence nanopore sensing. Experiments showing the polarity-dependence of event occurrence, and its frequency, as a function of $\mathrm{pH}$ showed the same qualitative behavior as for A1 in Supplementary Fig. 7, but with lower event frequencies overall. Both alginate samples were readily digested by alginate lyase (Supplementary Fig. 3) ${ }^{55}$, but infrared spectroscopy showed that A2 contained a dramatically greater proportion of carboxylate groups than A1 (Supplementary Fig. 8), so that the overall charge density of this molecule was expected to be higher than A1. Attempts to directly measure $\zeta$ potentials were complicated by corrosion of electrode surfaces, as noted in work examining the electrokinetics of protein transport through nanopores ${ }^{56}$. Further analysis was consistent with alginate A1 having a ratio of guluoronic $(\mathrm{G})$ to mannuronic (M) residues exceeding that of A2, with values from IR spectroscopy of $\sim 63 \% \mathrm{G} / 37 \% \mathrm{M}$ and $\sim 57 \% \mathrm{G} / 43 \% \mathrm{M}$, respectively ${ }^{49}$. Nanopore profiling of A2 showed differences compared to A1. Using the same electrolyte for $\mathbf{A} \mathbf{2}$ as for $\mathbf{A 1}$, measurements generated a $\sim$ sevenfold lower event frequency with longer durations for A2 compared to A1, in spite of the 75-fold higher A2 concentrations required for reasonable measurement times. Enzymatic digestion of $\mathbf{A} 2$ produced events at a higher frequency than for undigested A2, but still at lower frequency than for A1. The events for the digested sample of A2 were tenfold shorter-lived than for the A2 polymer, but not appreciably different in terms of blockage depth (Fig. 3, Supplementary Fig. 4). Measurements of the molecular-weight dependence of hyaluronic acid translocation through $\mathrm{He}$-ion-drilled $\mathrm{SiN}_{\mathrm{x}}$ nanopores showed $>10-100$-fold differences in event duration between $\sim 50$ and $2500 \mathrm{kDa}$ species, where the differences were expected to arise from macromolecular size, alone. The attenuation of shortduration events due to instrument response characteristics has been detailed in earlier work, and is accommodated here through the use of suitable fitting functions ${ }^{57,58}$. The standalone reliability of extended measurements using the same $\sim 10 \mathrm{~nm}$ pore for the alginate samples in series was limited by frequent partial pore clogging by $\mathbf{A} 2$ that occasionally led to long-lived partial blockages that required cleaning steps that could change the apparent nanopore diameter by up to $3 \mathrm{~nm}$. Nevertheless, these measurements using single pores yielded $f_{\mathrm{b}}$, $\tau$, and event frequency characteristics for the three analytes consistent with the results in Fig. 3. 
Characterization of clinical and contaminated heparin. These initial survey experiments showed measurement outcomes with strong sensitivity to analyte identity, with the number of anionic carboxylate moieties being a compelling differentiator between A1 and A2. We then turned to the pressing specific challenge of (anionic) heparin sensing and (anionic) OSCS impurity detection. The first change, from the earlier work, was that the signal generation voltage polarity (in 1 and $4 \mathrm{M} \mathrm{KCl}$ ) now corresponded with the conventional electrophoretic direction for an anionic species. Acid digestion experiments akin to those in Supplementary Fig. 3 confirmed that heparin could translocate through the pore in response to an applied voltage. As with A1, heparin could be detected in $1 \mathrm{M} \mathrm{KCl}$ electrolyte, but the heparin event blockage magnitude and event frequency were both greater in $4 \mathrm{M} \mathrm{KCl}$, and so measurements were performed at this higher salt concentration (see Supplementary Fig. 9 for representative events and a heat map). Plots of event frequency versus heparin concentration were linear (Fig. 4), with a limit of detection of 0.379 USP heparin units $\mathrm{mL}^{-1}$ (in a $500 \mu \mathrm{L}$ well). In comparison, clinical dosage levels of $\sim 10^{4}$ units.day $^{-1}$ using $\sim 10^{3}$ units. $\mathrm{mL}^{-1}$ stock solutions are not uncommon. Heparin and alginate fingerprints differed in appearance from each other, but also through the profoundly different measurement configuration-opposite applied voltage polarity and fourfold higher electrolyte concentration for heparin-used to acquire them. We were more keenly interested, though, in whether an OSCS impurity in heparin could be detected. We performed measurements on unadulterated USP samples of either heparin or OSCS under identical experimental conditions. On the level of individual events, heparin and OSCS differed in their apparent interaction with the nanopore, with OSCS having a greater propensity to permanently block the pore unless a $\sim 1.3 \mathrm{~V}$ (so-called zap) pulse-a common approach leveraging the electrokinetic basis of analyte motion-was quickly applied when indications suggesting an impending permanent blockage arose. In addition, events associated with the heparin and OSCS samples differed appreciably in the current fluctuations during individual current blockages: OSCS current blockages exhibited $\sim 2-3 \times$ greater current noise, $\sigma$ $\left(f_{\mathrm{b}}\right)$, than heparin-induced events. Overall, in spite of considerable overlap in the most frequent event $f_{\mathrm{b}}$ and $\tau$, the distribution of event characteristics revealed a key difference between heparin and OSCS samples (Fig. 5 and Supplementary Fig. 10). Namely, events measured using heparin samples exhibited a longer duration tail in the total event duration distribution, while events measured using OSCS samples exhibited a longer tail in $f_{\mathrm{b}}$. Measurements of mixtures of heparin and OSCS (16 ppm each) yielded event distributions showing both tails, consistent with the presence of both the heparin therapeutic and its contaminant. We developed an automatic thresholding procedure based on event distribution statistics in $f_{\mathrm{b}}$ and $\tau$ (details in the Supplementary Methods) to collapse the event distribution fingerprints into recognition flags denoting the presence or absence of each component. In brief, OSCS was declared present when events occurred with $f_{\mathrm{b} \text {,sample }} \lesssim \operatorname{mode}\left(f_{\mathrm{b}, \text { USP heparin }}^{\text {binned }}\right)-3 \sigma\left(f_{\mathrm{b}, \text { USP heparin }}^{\text {binned }}\right)$ and heparin was declared present when events occurred with $\tau_{\text {sample }} \gtrsim \operatorname{mode}\left(\left(\log _{10} \tau_{\text {USP OSCS }}\right)^{\text {binned }}\right)-3 \sigma\left(\left(\log _{10} \tau_{\text {USP OSCS }}\right)^{\text {binned }}\right)$. Figure 5 shows the correct recognition of USP heparin, USP OSCS, and a mixture of both, across four trials using nanopores of slightly different sizes. The OSCS contaminant levels detected here were fourfold lower (without efforts to explore a lower bound) than the OSCS detection limit reported in the work that examined and quantified the contaminant in suspect heparin lots ${ }^{18}$.

\section{Discussion}

We demonstrated the feasibility of using $\operatorname{SiN}_{\mathrm{x}}$ nanopores to characterize glycans exhibiting a variety of chemical

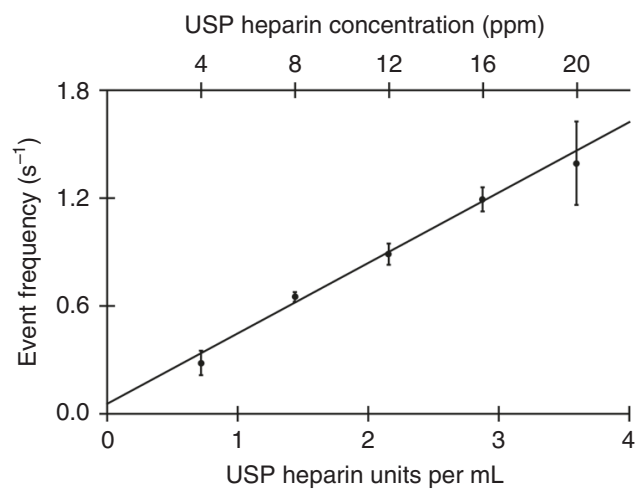

Fig. 4 Heparin calibration curve. Three trials were performed, with at least 500 events per run extracted from 900 s-long measurements in a $\sim 9.3 \mathrm{~nm}$ pore at $+200 \mathrm{mV}$ applied voltage difference after consecutive addition of $1 \mu \mathrm{L}$ aliquots to the headstage side of the same nanopore. Error bars are the standard deviation for the three trials

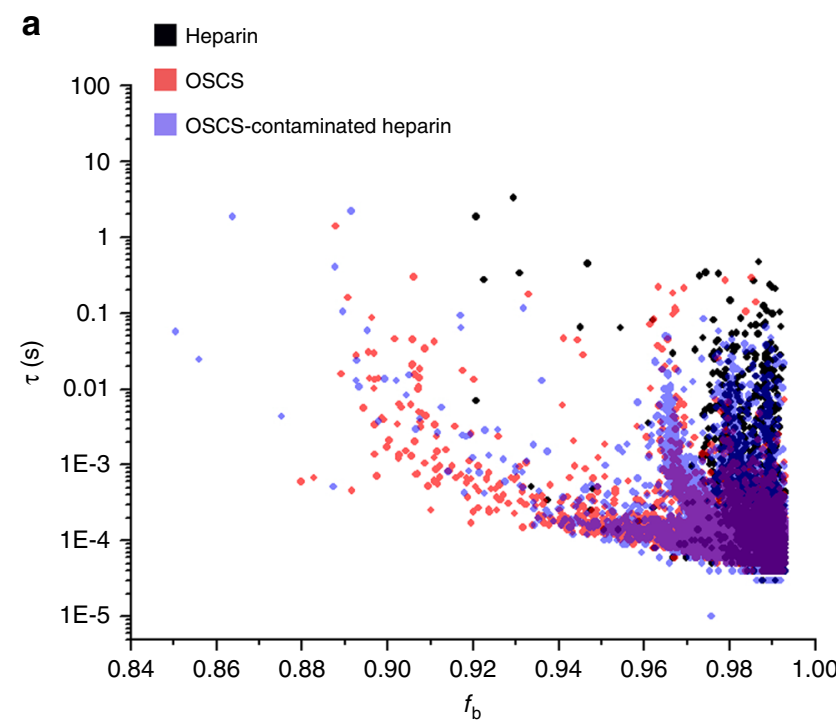

b
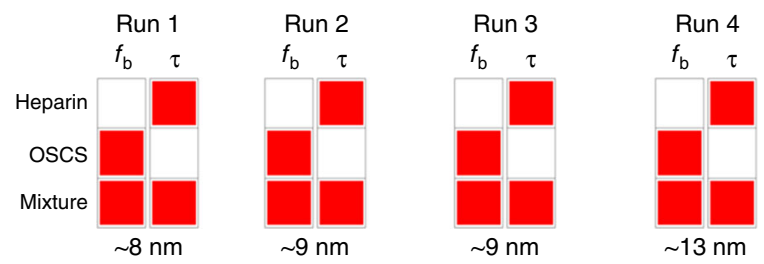

Fig. 5 Nanopore resistive-pulse analysis of heparin, OSCS, and their mixture. a Superimposed scatter plots of $16 \mathrm{ppm}$ heparin, OSCS and OSCScontaminated heparin added to $4 \mathrm{M}$ potassium chloride at $+200 \mathrm{mV}$ and measured using a $\sim 13 \mathrm{~nm}$ pore. The colors in the legend correspond to the listed sample, and are blended (using transparency) in the plot where events from different samples overlap. b Recognition flags of heparin, OSCS and their mixture from four independent trials accurately identify the presence of the OSCS aliquot in the mixture. The red squares denote recognition of a species from the distribution of the corresponding property (column)

compositions, including a prevalent therapeutic, heparin. The extremely high-charge density carried by heparin poses a particular challenge to a nanoscale sensor element that can, itself, be charged. More generally, unwanted interactions between analyte and nanopore-and the ease and feasibility of ameliorative steps-can imperil nanopore-based experiments: that none of 
the diverse polysaccharides considered here catastrophically clogged the nanopore-even when subjected to the stringent test of translocation through the pore-was salutary ${ }^{48}$. Indeed, nanopore sensing was successful over a number of electrolyte concentration ranges, from 0.1 to $4 \mathrm{M} \mathrm{KCl}$, for which shielding of the charged nanopore surface would be quite different in degree. With translocation possible through $\mathrm{SiN}_{\mathrm{x}}$ nanopores, even with their charged surface, a rich set of nanopore-based sensing configurations should be within reach. In this work, we used a straightforward resistive-pulse sensing paradigm to readily detect and differentiate between different polysaccharides, including enzymatic digestion products and two separate alginate samples differing in relative monomer composition. We used voltage polarity and electrolyte composition alongside the distribution of events as a function of $f_{\mathrm{b}}$ and $\tau$ to construct fingerprints and recognition flags characteristic of each sample. Linear calibration curves show that these measurements easily support concentration determinations in addition to analyte recognition.

From a fundamental perspective, nanopores can be a powerful tool for exploring molecular, interfacial, and intermolecular phenomena, often arising from only simple changes of experimental conditions. Electrolyte-dependent interfacial interactions -at nanopore and molecule surfaces-are complex, and treatments of widely varying levels of sophistication have emerged from decades of experimental and theoretical studies of the canonical nanopore-DNA system, in particular ${ }^{32}$. For example, changes of electrolyte concentration have been observed to reverse the sign of the current perturbation in DNA translocations through solid-state nanopores, and to decrease dextran sulfate blockage frequencies while increasing their durations using $\sim 1.3 \mathrm{~nm}$-diameter pores where the Debye length was comparable to the pore dimensions ${ }^{42,59}$. With the larger pores used here, overlapping Debye layers would not be expected in $0.1 \mathrm{M} \mathrm{KCl}$ solutions, leaving three expected principal effects of lowering the electrolyte concentration from $1 \mathrm{M} \mathrm{KCl}$ : a lowering of the potential across the pore and thus of the overall electrophoretic force on an analyte near the pore; a reduction in the available number of bulk ions displaced by the analyte volume; and a change in the ion distribution around charged interfacesthe nanopore and analyte surfaces-that influences the nanopore signal through a complex overall mechanism within a given experimental configuration. Blockage magnitudes measured here in the more conventional $1 \mathrm{M} \mathrm{KCl}$ would be consistent with, in a simple volume exclusion sense $\left(r_{\text {analyte }}^{2} / r_{\text {pore }}^{2}=1-f_{b}\right)$, translocation of linearized polysaccharides. Deeper blockages would be expected from the polysaccharides here with hydrodynamic radii on par with the nanopore diameters (for example, A1 and A2 have viscosity-derived free-solution hydrodynamic radii of $\sim 19$ and $\sim 8 \mathrm{~nm}$ ). Polysaccharide translocation was independently confirmed and signals were detected only when the analytes had access to the nanopores, so these recorded events either arose from analyte interactions with the pore mouth rather than from translocation, or the blockage magnitude analysis must include additional factors such as charge density carried by the analyte, itself, and consider mobile charges at the analyte-solution and solution-nanopore interfaces 59,60 . The effects of these and more complex interfacial phenomena emerged in one of the more unexpected observations in this work: that the voltage polarity for signal generation with both alginate samples was opposite to that expected for electrophoretic motion of an anionic polymer (for example, hyaluronic acid in $\mathrm{He}$-ion-drilled $\mathrm{SiN}_{\mathrm{x}}$ pores ${ }^{43}$ ), and the more charge-rich $\mathbf{A 2}$ was detected at a lower event frequency than A1. The voltage polarity contrasted with the electrophoretic polarity for detecting highly charge-dense, anionic heparin. Nanopore-based studies with polyethylene glycol polymers point to a change of effective analyte charge by sorption of electrolyte ions $\left(\mathrm{K}^{+}\right.$for those studies) with the resultant analyte motion then being electrophoretic for the voltage polarity and the sign of the sorbed charge ${ }^{29}$. The results of Supplementary Fig. 7, however, point to $\mathrm{pH}$-dependent changes in the voltage polarity required for sensing alginates, with the polarity having opposite signs on either side of the isoelectric point of $\mathrm{SiN}_{\mathrm{x}}$. Mirroring this change in the voltage polarity is the $\mathrm{SiN}_{\mathrm{x}}$ surface charge that is positive at lower $\mathrm{pH}$ and negative at higher $\mathrm{pH}^{45,46,56}$. This change in nanopore surface charge sign causes a reversal in the direction of electroosmotic motion for a fixed voltage polarity (and thus fixed electrophoretic direction). In addition, when an analyte contains at least one ionizable moiety (with associated $\mathrm{pK}_{\mathrm{a}}$ ), then changes of solution $\mathrm{pH}$ can also affect the analyte charge sign and density -and thus the voltage polarity required for electrophoresis in a given direction. The apparent mobility of an analyte in response to electrolyte flow through the surface-charged nanochannel is the sum of its electrophoretic and electroosmotic mobilities, which can both be tuned by the solution $\mathrm{pH}$. Work examining the electrokinetics of protein transport through silicon nitride nanopores showed that electroosmosis could overwhelm electrophoresis as the effect determining the direction of analyte motion ${ }^{56}$. Given the acidic functional groups in the analytes here, the changes in nanopore surface chemistry should dominate the effective mobility and its voltage polarity dependence. The event frequency and voltage polarity behaviors are consistent with the distinct physicochemical properties of each analyte with both electrophoresis and electroosmosis occuring simultaneously. In the negatively charged $\mathrm{SiN}_{\mathrm{x}}$ pores at $\mathrm{pH} \sim 7$, electroosmosis and electrophoresis are in opposition for anionic $\mathbf{A 1}$ and $\mathbf{A 2}$, and signal was generated in the electroosmotic direction for both. The electrophoretic force would be greater on the more highly charged A2, lowering its detection frequency in the opposing electroosmotic direction, relative to $\mathbf{A 1}$, consistent with observation. More detailed exploration of the differences between A1 and $\mathbf{A} 2$ must also contend with their different molecular weights and their different chain flexibilities arising from their different $\mathrm{M} / \mathrm{G}$ ratios. In the case of heparin, the charge density is sufficiently high so that events are detected using a voltage polarity that would electrophoretically drive the anionic polymer towards the nanopore. Experimental investigations including and beyond the ones presented here, exploring the underpinnings of the nanopore-generated signal using (polysaccharide) biopolymers such as these, with greater chemical and structural complexity than the canonical nanopore test molecule, DNA, or than homopolymers such as polyethylene glycol, should also provide fertile ground for high-level simulations. Interfacial effects will require additional study in the context of polysaccharides, but hold possibilities for tuning sensing selectivity and sensitivity. Indeed, explicit consideration of sensing conditions-including nanopore size, electrolyte composition, and voltage polarityalready augments the ability to compare nanopore molecular fingerprints as shown in Fig. 3.

The failure in 2008 to detect an OSCS contaminant in clinical heparin samples had previously led to patient morbidity and mortality ${ }^{14-18}$, so that our ability to use a simple nanopore-based assay to quantify heparin levels and detect OSCS at clinically meaningful contamination levels, is itself significant. In a broader sense, we expect that these initial results exploring polysaccharide structure can, by analogy with earlier nanopore DNA and protein sensing supporting genomics and proteomics, spotlight the potential of using nanopores as a tool for glycomics. The demonstration of polysaccharide translocation through nanofabrication-compatible $\mathrm{SiN}_{\mathrm{x}}$ nanopores portends the development of more sophisticated sensing schemes as seen in the use of nanopores for genomics. Similarly, the successful use of chemical tuning-of electrolyte composition and by enzyme addition 
- to alter the nanopore signal generated by diverse polysaccharides suggests that nanopore glycomics might borrow from and extend upon similar approaches developed for nanopore genomics. There is an ongoing need in glycomics for new tools to cope with the analytical challenges caused by the structural and physicochemical complexity of polysaccharides, and by the often inherently heterogenous nature of naturally derived carbohydrates. The demonstrations of nanopore sensing here provide a beachhead for ongoing efforts to develop solid-state nanopores as a promising platform technology for glycomics.

\section{Methods}

Nanopore formation and characterization. A full listing of the experimental details is available in the Supplementary Information. Nanopores were formed via controlled dielectric breakdown ${ }^{44}$ in nominally $10 \mathrm{~nm}$-thick silicon nitride $\left(\mathrm{SiN}_{\mathrm{x}}\right)$ membranes. Apparent nanopore sizes were inferred from their conductance, $G$, determined from Ohmic current-voltage data. This method for determining the size of a single nanopore has long-standing use, but has gained greater prominence with the emergence of low-overhead nanopore fabrication methods such as various implementations of the dielectric breakdown method ${ }^{44,61,62}$. Conductance-based characterization provides effective characterization of nanopores without the burdens of charged-particle microscope use, and so we adopted the approach within its conventional application framework. ${ }^{44-46,58,59,63-67}$. Nanopores used for measurements produced stable open-pore (analyte-free) currents in the electrolyte solutions used.

Polysaccharide profiling. Polysaccharides were commercially obtained: sodium alginate samples from two different sources-A1 (Alfa Aesar, Ward Hill, MA) and A2 (FMC Corporation Health and Nutrition, PA, USA); USP heparin sodium salt; and USP OSCS. For routine measurements, sample aliquots were added to the headstage side (Fig. 1), leaving the ground side free of initially added analyte. Current blockages were extracted using a current-threshold analysis. The experiment was configured as shown in Fig. 1, and applied voltage differences $\Delta V=$ $-V_{\text {headstage electrode }}$ were reported so that a positive value would be required for electrophoretic passage of an anionic molecule through the nanopore.

Data availability. The authors declare that the data supporting the findings of this study are available within the paper and its Supplementary Information files.

Received: 6 November 2017 Accepted: 17 July 2018

Published online: 16 August 2018

\section{References}

1. Imberty, A. \& Pérez, S. Structure, conformation, and dynamics of bioactive oligosaccharides: theoretical approaches and experimental validations. Chem. Rev. 100, 4567-4588 (2000).

2. DeMarco, M. L. \& Woods, R. J. Structural glycobiology: a game of snakes and ladders. Glycobiology 18, 426-440 (2008).

3. Dove, A. The bittersweet promise of glycobiology. Nat. Biotechnol. 19, 913-917 (2001).

4. Ernst, B. \& Magnani, J. L. From carbohydrate leads to glycomimetic drugs. Nat. Rev. Drug. Discov. 8, 661-677 (2009).

5. Pinho, S. S. \& Reis, C. A. Glycosylation in cancer: mechanisms and clinical implications. Nat. Rev. Cancer 15, 540-555 (2015).

6. Seeberger, P. H. Chemical glycobiology: why now? Nat. Chem. Biol. 5, 368-372 (2009).

7. Lichtenstein, R. G. \& Rabinovich, G. A. Glycobiology of cell death: when glycans and lectins govern cell fate. Cell Death Differ. 20, 976-986 (2013)

8. Dalziel, M., Crispin, M., Scanlan, C. N., Zitzmann, N. \& Dwek, R. A. Emerging principles for the therapeutic exploitation of glycosylation. Science 343, 1235681 (2014).

9. Varki, A et al. Essentials of Glycobiology. 2nd edn, (Cold Spring Harbor Laboratory Press, Cold Spring Harbor (NY), 2009).

10. Linhardt, R. J. \& Toida, T. Role of glycosaminoglycans in cellular communication. Acc. Chem. Res. 37, 431-438 (2004)

11. Kovalenko, I. et al. A major constituent of brown algae for use in highcapacity Li-ion batteries. Science 334, 75-79 (2011).

12. National Research Council. Transforming Glycoscience: A Roadmap for the Future. (The National Academies Press, Washington DC, 2012).

13. Czjzek, M. Biochemistry: a wine-induced breakdown. Nature 544, 45-46 (2017).
14. Lester, J., Chandler, T. \& Gemene, K. L. Reversible electrochemical sensor for detection of high-charge density polyanion contaminants in heparin. Anal. Chem. 87, 11537-11543 (2015).

15. Kim, D.-H., Park, Y. J., Jung, K. H. \& Lee, K.-H. Ratiometric detection of nanomolar concentrations of heparin in serum and plasma samples using a fluorescent chemosensor based on peptides. Anal. Chem. 86, 6580-6586 (2014).

16. Liu, H., Zhang, Z. \& Linhardt, R. J. Lessons learned from the contamination of heparin. Nat. Prod. Rep. 26, 313-321 (2009).

17. Korir, A. \& Larive, C. Advances in the separation, sensitive detection, and characterization of heparin and heparan sulfate. Anal. Bioanal. Chem. 393, 155-169 (2009)

18. Kishimoto, T. K. et al. Contaminated heparin associated with adverse clinical events and activation of the contact system. New Engl. J. Med 358, 2457-2467 (2008).

19. Guerrini, M. et al. Oversulfated chondroitin sulfate is a contaminant in heparin associated with adverse clinical events. Nat. Biotech. 26, 669-675 (2008).

20. Kailemia, M. J., Ruhaak, L. R., Lebrilla, C. B. \& Amster, I. J. Oligosaccharide analysis by mass spectrometry: a review of recent developments. Anal. Chem. 86, 196-212 (2014).

21. Kasianowicz, J. J., Brandin, E., Branton, D. \& Deamer, D. W. Characterization of individual polynucleotide molecules using a membrane channel. Proc. Natl Acad. Sci. USA 93, 13770-13773 (1996)

22. Haywood, D. G., Saha-Shah, A., Baker, L. A. \& Jacobson, S. C. Fundamental studies of nanofluidics: nanopores, nanochannels, and nanopipets. Anal. Chem. 87, 172-187 (2015).

23. Taniguchi, M. Selective multidetection using nanopores. Anal. Chem. 87, 188-199 (2015)

24. Reiner, J. E. et al. Disease detection and management via single nanoporebased sensors. Chem. Rev. 112, 6431-6451 (2012).

25. Howorka, S. \& Siwy, Z. Nanopore analytics: sensing of single molecules Chem. Soc. Rev. 38, 2360-2384 (2009).

26. Miles, B. N. et al. Single molecule sensing with solid-state nanopores: novel materials, methods, and applications. Chem. Soc. Rev. 42, 15-28 (2013).

27. Oukhaled, A., Bacri, L., Pastoriza-Gallego, M., Betton, J.-M. \& Pelta, J. Sensing proteins through nanopores: fundamental to applications. Acs. Chem. Biol. 7, 1935-1949 (2012)

28. Branton, D. et al. The potential and challenges 6 of nanopore sequencing. Nat Biotechnol. 26, 1146-1153 (2008).

29. Reiner, J. E., Kasianowicz, J. J., Nablo, B. J. \& Robertson, J. W. F. Theory for polymer analysis using nanopore-based single-molecule mass spectrometry. Proc. Natl Acad. Sci. USA 107, 12080-12085 (2010).

30. Wanunu, M., Sutin, J., McNally, B., Chow, A. \& Meller, A. DNA translocation governed by interactions with solid-state nanopores. Biophys. J. 95, 4716-4725 (2008).

31. Carbonaro, A. \& Sohn, L. L. A resistive-pulse sensor chip for multianalyte immunoassays. Lab. Chip. 5, 1155-1160 (2005).

32. Aksimentiev, A. Deciphering ionic current signatures of DNA transport through a nanopore. Nanoscale 2, 468-483 (2010).

33. Bacri, L. et al. Discrimination of neutral oligosaccharides through a nanopore. Biochem. Biophys. Res. Commun. 412, 561-564 (2011).

34. Fennouri, A. et al. Single molecule detection of glycosaminoglycan hyaluronic acid oligosaccharides and depolymerization enzyme activity using a protein nanopore. ACS Nano. 6, 9672-9678 (2012).

35. Fennouri, A. et al. Kinetics of enzymatic degradation of high molecular weight polysaccharides through a nanopore: experiments and data-modeling. Anal. Chem. 85, 8488-8492 (2013).

36. Kullman, L., Winterhalter, M. \& Bezrukov, S. M. Transport of maltodextrin through maltoporin: a single-channel study. Biophys. J. 82, 803-812 (2002).

37. Zhao, S. et al. Sugar-stimulated robust nanodevice: 4-carboxyphenylboronic acid modified single glass conical nanopores. Electrochem. Commun. 36, 71-74 (2013)

38. Zheng, Y.-B. et al. A temperature, $\mathrm{pH}$ and sugar triple-stimuli-responsive nanofluidic diode. Nanoscale 9, 433-439 (2017).

39. Nguyen, Q. H., Ali, M., Neumann, R. \& Ensinger, W. Saccharide/glycoprotein recognition inside synthetic ion channels modified with boronic acid. Sens. Actuat B-Chem. 162, 216-222 (2012).

40. Vilozny, B. et al. Carbohydrate-actuated nanofluidic diode: switchable current rectification in a nanopipette. Nanoscale 5, 9214-9221 (2013).

41. Sun, Z. et al. pH gated glucose responsive biomimetic single nanochannels. Chem. Commun. 48, 3282-3284 (2012).

42. Oukhaled, G., Bacri, L., Mathé, J., Pelta, J. \& Auvray, L. Effect of screening on the transport of polyelectrolytes through nanopores. EPL (Europhys. Lett.) 82, 48003 (2008).

43. Rivas, F. et al. Label-free analysis of physiological hyaluronan size distribution with a solid-state nanopore sensor. Nat. Commun. 9, 1037 (2018). 
44. Kwok, H., Briggs, K. \& Tabard-Cossa, V. Nanopore fabrication by controlled dielectric breakdown. PLoS One 9, e92880 (2014).

45. Dwyer J. R., Bandara YMNDY, Whelan J. C., Karawdeniya B. I., Nichols J. W. in Nanofluidics, 2nd edn (eds Edel J., Ivanov A., Kim M.) (Royal Society for Chemistry, London, 2016).

46. Dwyer, J. R. \& Harb, M. Through a window, brightly: a review of selected nanofabricated thin-film platforms for spectroscopy, imaging, and detection. Appl. Spectrosc. 71, 2051-2075 (2017).

47. Im, J. et al. Electronic single-molecule identification of carbohydrate isomers by recognition tunnelling. Nat. Commun. 7, 13868 (2016).

48. Yusko, E. C. et al. Controlling protein translocation through nanopores with bio-inspired fluid walls. Nat. Nanotechnol. 6, 253-260 (2011).

49. Pereira, L., Sousa, A., Coelho, H., Amado, A. M. \& Ribeiro-Claro, P. J. A. Use of FTIR, FT-Raman and 13C-NMR spectroscopy for identification of some seaweed phycocolloids. Biomol. Eng. 20, 223-228 (2003).

50. Linhardt, R. J. 2003 Claude S. Hudson Award address in carbohydrate chemistry. Heparin: structure and activity. J. Med. Chem. 46, 2551-2564 (2003).

51. Viskov, C. et al. Isolation and characterization of contaminants in recalled unfractionated heparin and low-molecular-weight heparin. Clin. Appl. Thromb.-Hem. 15, 395-401 (2009).

52. Foulger, J. H. The use of the Molisch ( $\alpha$-naphthol) reactions in the study of sugars in biological fluids. J. Biol. Chem. 92, 345-353 (1931).

53. Hallal, J. L. J., Lucho, A. M. S. \& Gonçalves, R. S. Electrochemical polymerization of furfural on a platinum electrode in aqueous solutions of potassium biphthalate. Mat. Res 8, 23-29 (2005).

54. Hoogerheide, D. P., Garaj, S. \& Golovchenko, J. A. Probing surface charge fluctuations with solid-state nanopores. Phys. Rev. Lett. 102, 256804 (2009).

55. Skidmore, M. A., Guimond, S. E., Dumax-Vorzet, A. F., Yates, E. A. \& Turnbull, J. E. Disaccharide compositional analysis of heparan sulfate and heparin polysaccharides using UV or high-sensitivity fluorescence (BODIPY) detection. Nat. Protoc. 5, 1983-1992 (2010).

56. Firnkes, M., Pedone, D., Knezevic, J., Döblinger, M. \& Rant, U. Electrically facilitated translocations of proteins through silicon nitride nanopores: conjoint and competitive action of diffusion, electrophoresis, and electroosmosis. Nano Lett. 6, 895--8909 (2010).

57. Uram, J. D., Ke, K. \& Mayer, M. Noise and bandwidth of current recordings from submicrometer pores and nanopores. ACS Nano. 2, 857-872 (2008).

58. YMNDY, Bandara, Nichols, J. W., Iroshika Karawdeniya, B. \& Dwyer, J. R. Conductance-based profiling of nanopores: accommodating fabrication irregularities. Electrophoresis 39, 626-634 (2018).

59. Smeets, R. M. M. et al. Salt dependence of ion transport and DNA translocation through solid-state nanopores. Nano. Lett. 6, 89-95 (2006).

60. Binquan, L. \& Aleksei, A. Control and reversal of the electrophoretic force on DNA in a charged nanopore. J. Phys. Condens. Matter 22, 454123 (2010).

61. Autumn, T. C., Kyle, B., Adam, R. H. \& Vincent, T.-C. Solid-state nanopore localization by controlled breakdown of selectively thinned membranes. Nanotechnology 28, 085304 (2017).

62. Goto, Y., Yanagi, I., Matsui, K., Yokoi, T. \& Takeda, K-i. Integrated solid-state nanopore platform for nanopore fabrication via dielectric breakdown, DNAspeed deceleration and noise reduction. Sci. Rep.-UK 6, 31324 (2016).

63. Liebes, Y. et al. Reconstructing solid state nanopore shape from electrical measurements. Appl. Phys. Lett. 97, 223105 (2010).

64. YMNDY, Bandara, Karawdeniya, B. I. \& Dwyer, J. R. Real-time profiling of solid-state nanopores during solution-phase nanofabrication. ACS Appl. Mater. Interfaces 8, 30583-30589 (2016).
65. Frament, C. M., Bandara, N. \& Dwyer, J. R. Nanopore surface coating delivers nanopore size and shape through conductance-based sizing. ACS Appl. Mater. Interfaces 5, 9330-9337 (2013).

66. Frament, C. M. \& Dwyer, J. R. Conductance-based determination of solidstate nanopore size and shape: an exploration of performance limits. J. Phys. Chem. C. 116, 23315-23321 (2012).

67. Lee, C. et al. Large apparent electric size of solid-state nanopores due to spatially extended surface conduction. Nano. Lett. 12, 4037-4044 (2012).

\section{Acknowledgements}

This research has been supported by NSF CAREER award CBET-1150085, and by the University of Rhode Island, including URI graduate fellowships for Y. M. Nuwan D. Y Bandara and Buddini Iroshika Karawdeniya. We thank Travis Leffert for preliminary polysaccharide measurements. We thank the Lucht Group (URI Dept. of Chemistry) for access to their IR spectrometer, and K.W.D. Kaveendi Chandrasiri and Bharathy S. Parimalam for acquiring IR spectra. We thank the group of Prof. Stephen Kennedy (URI Depts. of Electrical, Computer and Biomedical Engineering, \& Chemical Engineering) for samples of alginate $\mathbf{A 2}$.

\section{Author contributions}

B.I.K. developed protocols for and carried out nanopore polysaccharide sensing, carried out control and characterization studies, and performed data analysis. Y.M.N.D.Y.B wrote custom data analysis software and performed heparin and OSCS studies with B.I.K. in addition to characterization studies. J.W.N. conducted the initial heparin and OSCS measurements and optimized sensing conditions. R.B.C developed the digestion-based optical characterization measurements. J.R.D proposed and guided the work. All authors have contributed to, and approve, the manuscript.

\section{Additional information}

Supplementary Information accompanies this paper at https://doi.org/10.1038/s41467018-05751-y.

Competing interests: The authors declare no competing interests.

Reprints and permission information is available online at http://npg.nature.com/ reprintsandpermissions/

Publisher's note: Springer Nature remains neutral with regard to jurisdictional claims in published maps and institutional affiliations.

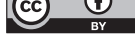

Open Access This article is licensed under a Creative Commons Attribution 4.0 International License, which permits use, sharing, adaptation, distribution and reproduction in any medium or format, as long as you give appropriate credit to the original author(s) and the source, provide a link to the Creative Commons license, and indicate if changes were made. The images or other third party material in this article are included in the article's Creative Commons license, unless indicated otherwise in a credit line to the material. If material is not included in the article's Creative Commons license and your intended use is not permitted by statutory regulation or exceeds the permitted use, you will need to obtain permission directly from the copyright holder. To view a copy of this license, visit http://creativecommons.org/ licenses/by/4.0/.

(C) The Author(s) 2018 